\title{
D-Medium Control the Temperature of Electron Inside and Outside the Atom
}

\author{
Saddam Husain Dhobi, Roshan Subedi, Keshab Ghimire, Deepak Kharel, Sudeep Ghimire
}

\begin{abstract}
The objective of this work is to show the presence of D medium and the presence of this medium to control the temperature of an electron inside or outside the atom. For this, some mathematical model developed in [1], work for temperature with velocity was developed and presence of D-Medium inside atom described [2]. On interconnecting temperature and $D$-Medium, the concept of D-medium generated and shows that D-medium surrounded the electron in all cases (free, inside an atom, or other), this medium controls the temperature of the electron. Hence temperature of an electron depends upon the D-medium either inside an atom or outside the atom. The penetration of a specific photon through this medium also increases the temperature of the electron because the electron is composed of sub-electronic particles [3].

Keywords: D-Medium, Temperature, Electron, Sub-Electronic Particles, Inside and Outside, etc.
\end{abstract}

\section{INTRODUCTION AND BACKGROUND}

The interaction of a photon with an electron gives the phenomena of energy transformation for a photon to electron and this phenomenon required a certain time. This time of transformation of energy to the electron depends upon the energy of a photon that is if the photon has low energy the transformation of energy time to the electron is larger while if the photon has high energy the time transformation of energy to the electron is less. This time different transformation of time shows electron is consentient of sub-electronic particles fermion, bosons and D-particles [3].

After the absorption of energy by an electron from the photon, the constituent particles vibrate and disturbed the size of the electron, therefore, the size of the electron also depends upon the incidence of photon energy on it [4]. The compassion of cross-section area of photon and electron and theoretical model [5], and found that surface area of a single electron is very small than that of a photon. So when the

Manuscript received on 29 March 2021 | Revised Manuscript received on 03 April 2021 | Manuscript Accepted on 15 April 2021 | Manuscript published on 30 April 2021.

* Correspondence Author

Saddam Husain Dhobi*, Department of Physics, Patan Multiple Campus, Lalitpur, Tribhuvan University, Nepal. Email: saddam@ran.edu.np

Roshan Subedi, Department of Physics, Patan Multiple Campus, Lalitpur, Tribhuvan University, Nepal. Email: rosansubedi17@gmail.com

Keshab Ghimire, Department of Physics, Patan Multiple Campus, Lalitpur, Tribhuvan University, Nepal. Email: kghimire805@gmail.com

Deepak Kharel, Department of Physics, Golden Gate International College, Tribhuvan University, Kathmandu-44600, Nepal. Email:deepak.kharel2049@gmail.com

Sudeep Ghimire, Department of Physics, Patan Multiple Campus, Lalitpur, Tribhuvan University, Nepal. Email: sudeepghm46@gmail.com

(C) The Authors. Published by Lattice Science Publication (LSP). This is an open access article under the CC-BY-NC-ND license (http://creativecommons.org/licenses/by-nc-nd/4.0/)

photon of large cross-section area incidence on electron only absorbed the energy of those part of the photon which is in contact with it but not those part of photon energy which is a certain distance away from it. This phenomenon of photon show photon is a composition of tiny segment photon. This phenomenon is seen in cases of the photoelectric effect, Compton Effect, Raman Effect, and another type of matter-light interaction.

The existence of the D-medium inside an atom and its effectiveness on the speed of the electron inside the atom is studied [2], moreover, D-medium is a non- homogeneous transparent medium that plays an important role in the absorption of a photon and traveling the distance. The calculation of and comparison of the cross-section area of electron and photon (red) also shows the cross-section area of the red photon is greater than that of the electron. Moreover, found that the cross-section area of one photon is equal to approx. 650556.954 time's cross-section area of the electron [6].

\section{A. Kinetics energy of a particle in a particle accelerator experiment}

The CERN LHC operates up to $\mathrm{TeV}$ and has the limitation that only cosmic rays can show what lies beyond, the cosmic ray spectrum is expected to extend up to the Planck energy $\left(1.22 \times 10^{28} \mathrm{eV}\right.$, about $10^{15}$ times higher than the LHC). The Planck energy is energy expected for a vibrating string theory and is roughly 2 billion Joules [7]. This particle energy is the kinetic energy of the particle therefore the temperature of the necked electron is very and the D-Medium surrounding the electron balances it.

The size of the accelerating structure is the length of the drift tubes and this will protect the particles from the negative half-wave of the sinusoidal AC voltage. The required frequency for such length of the tube is applied radiofrequency (RF) voltage. The time-span of the negative half-wave is defined by the applied frequency,

$$
\begin{aligned}
& \left(\Delta t=\frac{\tau_{r f}}{2}\right) \quad \text { and length is } l_{n}=v_{n} \times \frac{\tau_{r f}}{2} \\
& l_{n}=\frac{1}{v_{r f}} \cdot \sqrt{\frac{n q U_{0} \sin \psi_{s}}{2 m}}
\end{aligned}
$$

which defines the design concept of the machine based on a device of the Unilac at the Institute for Heavy Ion Research (GSI) in Darmstadt, Germany, the kinetic energy of particle [8] in accelerators is given as,

$$
E_{\text {in }}=\frac{1}{2} m v^{2}
$$

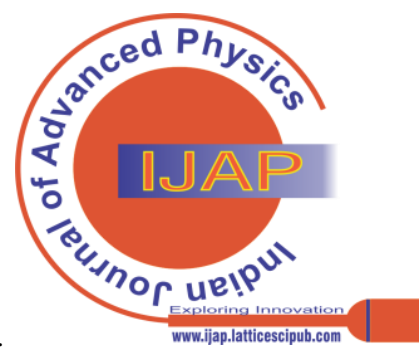


Basic Principles of all accelerators are based on the same principle that is a charged particle accelerates between a gap between two electrodes and Energy transferred to particles moving in between two electrodes is $E=Q V$. To control the temperature in the accelerator different coolant liquids or solid or other materials are used, for example like helium, water, and others [9].

\section{RESULT AND DISCUSSION}

\section{A. Temperature of the electron with kinetic energy}

The total energy of the electron in orbits energies is the sum of its kinetic and potential energy as

$$
\mathrm{E}_{\mathrm{n}}=\mathrm{K} \mathrm{E} \cdot \mathrm{P} \cdot \mathrm{E}
$$

with Kinetic energy

$$
K . E \cdot=\frac{1}{2} m_{e} v^{2}
$$

on assuming nonrelativistic motion and Potential energy with P.E. $=q_{e} V_{a}$ where $V$ is the potential due to the nucleus

$$
\text { as } V=\frac{k Z_{q_{\varrho}}}{r_{\mathbb{n}}}
$$

for charge qe. The total energy in stationary orbit is related as $E_{n}^{\prime}=-\frac{z^{2}}{n^{2}} E_{0}(n=1,2,3, \ldots)$

For the orbital energies of hydrogen-like atoms with $\mathrm{E}_{0}$ which is the ground-state energy $(n=1)$ for hydrogen $(Z=1)$ and calculated as

$$
E_{0}=\frac{2 \pi q_{0}^{4} m_{e} k^{3}}{k^{2}}=13.6 \mathrm{eV}
$$

\section{B. Case I: In absence of external energy}

The calculation of temperature at the different orbit of an electron for the hydrogen atom is derived with the help of kinetic energy of the electron and $\frac{3}{2} k_{B} T$ as

$$
T^{N E}=\frac{2}{3 k_{B}} \frac{\theta^{4} m m_{E}}{8 h^{2} \varepsilon_{0}^{2}} \frac{z^{2}}{n^{2}} .
$$

This is the temperature of the electron in the orbit of an atom without absorption of the energy from external sources [1].

C. Case II: In presence of external energy less the threshold Considering photoelectric effect, Case $I$ and $\frac{3}{2} k_{B} T$ temperature of the electron is observed for the hydrogen-like atom as, On putting the value of constant in the second term of equation (5), we get the temperature of the electron in orbit of the atom as

$$
T^{H L A}=\frac{2 h f}{3 k_{B}}+1.05258376 \times 10^{5}\left(\frac{z^{2}}{n^{2}}\right) .
$$

This temperature is the temperature for the electron that absorbed the energy from the incidence photon but the energy is not enough for photoelectric phenomena. In this phenomenon, the electron absorbed the energy and goes revolving around the nucleus of an atom. Therefore the kinetic energy of the electron is high than before the absorption of light. So, the temperature goes of the electron inside the atom goes to increases.

Table- I: Temperature of the electron in different orbit of the Hydrogen atom with zero external energy

\begin{tabular}{|c|c|c|c|l|l|}
\hline \multirow{2}{*}{ S.N. } & \multirow{2}{*}{$\mathbf{Z}$} & \multicolumn{4}{|c|}{ Temperature (K) } \\
\cline { 3 - 6 } & & $\mathbf{n = 1}$ & $\mathbf{n = 2}$ & \multicolumn{1}{|c|}{$\mathbf{n = 3}$} & \multicolumn{1}{|c|}{$\mathbf{n = 4}$} \\
\hline 1. & 1 & $1.05258376 \times 10^{5}$ & $0.26314594 \times 10^{5}$ & $0.11695375 \times 10^{5}$ & $0.06578648 \times 10^{5}$ \\
\hline 2. & 3 & $9.47325384 \times 10^{5}$ & $2.36831346 \times 10^{5}$ & $1.052558376 \times 10^{5}$ & $0.59207836 \times 10^{5}$ \\
\hline 3. & 11 & $127.362635 \times 10^{5}$ & $31.8406588 \times 10^{5}$ & $14.1514039 \times 10^{5}$ & $7.96016469 \times 10^{5}$ \\
\hline 4. & 19 & $379.982737 \times 10^{5}$ & $94.9956842 \times 10^{5}$ & $42.2203041 \times 10^{5}$ & $23.7489211 \times 10^{5}$ \\
\hline
\end{tabular}

D. Case III: In presence of external energy greater less than the threshold

If electron absorbed the energy $h f_{\text {ine }} \neq 0>$ threshold

then the electron either goes Photoelectric effect or ionization phenomena electron (accelerate the electron) and then case II can develop as

$$
T^{E}=\frac{2}{3 k_{B}} h f_{\text {ine }}-\frac{2}{3 k_{B}} \phi+T_{N E} .
$$

This is the temperature of the photoelectric effect electron. In this is the sum of the kinetic energy of the electron is the sum of the kinetic energy of revolving electron around the nuclease plus the absorbed energy. Consider the cases I, II and III the relation of the temperature of atomic electron it depends upon the $\mathrm{Z}$ and $\mathrm{n}$ for hydrogen and hydrogen-like atom. The temperature of the electron in orbit goes increases with $\mathrm{Z}$ and decreases with $\mathrm{n}$. when the electron absorbed energy then the temperature of the electron depends upon the incidence energy of the photon (external energy) in the case of the non-photoelectric effect (absorption of energy by electron) and the photoelectric effect. This means the temperature of electrons goes increase with the absorption of energy (external energy). The temperature listed in table 1 shows electron temperature is the order of $10^{5} \mathrm{~K}$ which is minimum this is because an electron in this condition doesn't observe energy and temperature is due to centrifugal and Columb force. Although the temperature is high when an atom with such temperature to human or any object no destruction or damage takes place.

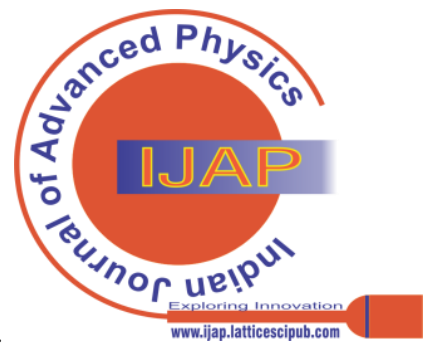


This destruction is due to the medium surrounding the electron that is the surrounding medium control the temperature and balance to normal temperature. The medium surrounded the electron is called D-Medium. This D-medium also has properties to balance or control the natural temperature and when the external temperature is applied the medium gets disturbed due to its composition.

\section{Free electron Temperature}

In the atmosphere, there are a large number of free electrons moving with high speed (approximate light velocity) and goes collide with an obstacle (atomic scale, microscopic and macroscopic) but the temperature carrying at such speed of electron doesn't affect. This is due to the presence of D-Medium which surrounds the electrons either inside an atom or in the Free State of electron or electron in a high-temperature environment. In a high-temperature environment D-Medium temperature increases and hence the temperature of electron increase. Therefore due to this D-Medium, the temperature of the electron in at natural state is normal (inside the atom and outside atom) while at external temperature environment D-Medium absorbed the heat and increase the temperature of the electron.

\section{E. Temperature of an electron at the different condition and} its control with D-Medium

Without external energy, external energy less than threshold energy (photoelectric effect does not take place), and external energy greater than threshold energy (photoelectric effect takes place).

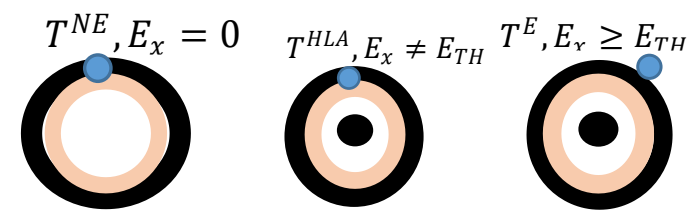

Figure 1: Temperature of the electron in hydrogen atom without external energy, with external energy less hen threshold and with external energy greater than the threshold

Here in figure $1, E_{x}$ is the external energy of the photon and $E_{T H}$ is threshold energy of the material, and the figure above states numbers of conditions at which the temperature of the electron is different. The left side figure show electron revolved around the nucleus in D-Medium when electron or atom doesn't absorb energy, the middle figure shows electron get external energy but energy is not sufficient to kick out the electron from the atom while the right figure shows electron absorbed energy from external show trying to go away from the atom. In the figure, D-medium is represented black and light orange, blue solid circle electron and black solid at the center is the nucleus.

\section{CONCLUSION}

The presence of D-Medium around or with electron control the temperature of the electron inside the atom and outside the atom. Inside the atom means electron presence in the orbital of the atom and due to the presence of D-Medium in an atom or surrounded the electron in an atom the temperature of an electron inside the atom doesn't go to exchange therefore the temperature of an electron in atom for different orbitals are different. Hence in this way, the temperature of an electron inside the atom is different from each other, and if these electrons are ejected by any means from an atom the temperature of the electron outside the atom is different because the medium goes to the external environment. That means the D-Medium of the electron in a free state depends upon the external environment. Therefore the existence of D-medium around or with electron controls the temperature of electron either inside an atom or outside the atom. Moreover, also depend upon the external temperature environment.

\section{ACKNOWLEDGMENT}

We would like to thanks all the members of the innovative Ghar Nepal and Robotics Academy of Nepal for providing research space and related facilities for this work. Similarly, we would also like to thanks all the members of the Department of physics, Patan Multiple Campus, Tribhuvan University, Nepal.

\section{REFERENCES}

1. B. Karki, S. H. Dhobi, K. Yadav, "Temperature of Electron Inside and Outside of Atom,” Technical Reports of Kansai University, vol.63, issu.3, 2020, p7483-7491

2. S. H. Dhobi, M. Neupane, S. Khanal, D. Dhakal, P. Shrestha, MD. J. Rangrej, "Theoretical Model for the Existence of a D- Medium inside an Atom," International Journal of Scientific \& Engineering Research, vol.10, issu.10, 2019, p.908-912.

3. S. H. Dhob, "Electron Take Different Time to Observe the Energy of Difference Photon," International Journal of Multidisciplinary Sciences and Advanced Technology, vol.1, issu.2, 2020, p.1-6.

4. S. H. Dhobi, MD. J. Rangrej, P. Bajagain, G. P. Adhikari, M. N. Upadhyay, R. P. Dahal, U. Patel, K. Ghimire, J. B. Baniya, S. K. Sharma, A. Panthi, "Shape and Size of Electron are Determine by the Amount of Photon Energy Incidence on It," Technical Reports of Kansai University, vol.62, issu.2, 2020, p.45-50.

5. S. H. Dhobi, P. Verma, P. Bajagain, MD. J. Rangrej, “Area Of Photon And Electron Are The Major Evidence To Causes Ejection Of One Electron By One Photon And Show Photon Is Composition," Global Scientific Journal, vol.7, Issu.11, 2019, p.664-667.

6. S. H. Dhobi, C. B. T. Lama, MD. J. Rangrej, T. R. Karki, Y. Limbu, S. Humagain, "Numerical Analysis of Photon and Electron Size, and Verifying One to One Correspond Rule of Einstein Photoelectric Effect," International Journal of Scientific \& Engineering Research vol. 10, Issu. 11, November, 2019, p.838-840

7. O. Frasciello, Basic principles of particle accelerator Physics, Seminar at University of Rome, Physics Department, February 9th, Italy, 2017, p.1-35.

8. B.J. Holzer, Introduction to Particle Accelerators and their Limitations, CERN: Geneva, Switzerland, 2016, p.23-29

9. N. R. Chevaliera, T. Junqueraa, J. P. Thermeaub , L. M. Romãoc, D. Vandeplasschec, "Cryogenic system for the MYRRHA superconducting linear accelerator," Advances in Cryogenic Engineering AIP Conf. Proc. 1573, 2014, p. 315-322. [CrossRef]

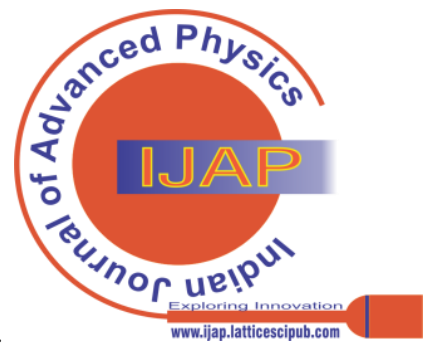




\section{AUTHORS PROFILE}

Mr. Saddam Husain Dhobi, pursed B. Sc., \& M. Sc., in Physical Science from Tribhuvan University, Kathmandu-44600, Nepal 2016, \& 2020. He is currently working as Sr. Researcher in Innovative Ghar Nepal Laboratory and Robotics Academy of Nepal, Lalitpur-44700, Nepal since 2013. He has published more than 37 research papers in reputed international journals including Thomson Reuters (SCI \& Web of Science), it's also available online. His research work focuses on electron and photon properties beyond the general properties, Data Analytics, Virology, Nuclear Physics, and Computational Intelligence based education. He has 4 years of research experience in related field.

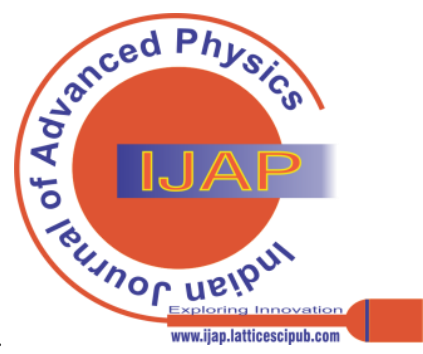

